\title{
Lipidomic Profiling of Mastoid Bone and Tissue from Patients with Chronic Otomastoiditis
}

\author{
Farbod Fazlollahi ${ }^{1}$ Kessiri Kongmanas ${ }^{2}$ Nongnuj Tanphaichitr ${ }^{2}$ Jeffrey Suh ${ }^{3}$ Kym Faull ${ }^{1}$ \\ Quinton Gopen ${ }^{3}$ \\ ${ }^{1}$ Department of Psychiatry and Biobehavioral Sciences, UCLA, Los \\ Angeles, United States \\ 2 Department of Biochemistry/Microbiology/Immunology, University \\ of Ottawa, Ottawa, Ontario, Canada

\begin{abstract}
Address for correspondence Farbod Fazlollahi, BS, Department of Psychiatry and Biobehavioral Sciences, UCLA, 760 Westwood Plaza, CHS 68-146, Los Angeles, CA 90095, United States (e-mail: farbodfazlollahi@gmail.com).
\end{abstract}

3 Department of Otorhinolaryngology-Head and Neck Surgery, UCLA Health System, Los Angeles, California, United States

Int Arch Otorhinolaryngol 2015;19:141-150.

\begin{abstract}
Introduction Chronic otomastoiditis causes pain, otorrhea, and hearing loss resulting from the growth of tissue within the normally hollow mastoid cavity.

Objectives In this report, we used a lipidomics approach to profile major mastoid bone and tissue lipids from patients with and without otomastoiditis.

Methods The bone dust created during mastoidectomy, as well as the mastoid tissue, was analyzed from seven patients. Bone dust was also collected and analyzed in an additional four otologic cases (parotidectomy requiring mastoidectomy). Samples were subjected to a modified Bligh/Dyer lipid extraction, then high-performance thin-layer chromatography (HPTLC), combined gas chromatography/electron impact-mass spectrometry (GC/El-MS), and flow-injection/electrospray ionization-tandem mass spectrometry (FI/ESI-MSMS). Data were analyzed for identification and profiling of major lipid components.

Results HPTLC revealed the presence of various lipid classes, including phosphatidylcholines, cholesterol, and triacylglycerols. GC/EI-MS analysis revealed the presence of cholesterol and several fatty acids. FI/ESI-MSMS analysis revealed a host of phosphatidylcholines, phosphatidylethanolamines, and cholesteryl esters.

Conclusion We used a lipidomics approach to develop an efficient (both in time and tissue amount) methodology for analysis of these tissues, identify the most abundant

Keywords

- mastoiditis

- lipid metabolism

- mass spectrometry

- flow injection analysis and common lipid species, and create a base of knowledge from which more focused endeavors in biomarker discovery can emerge. In an effort toward improved patient categorization and individualized intervention, the ultimate goal of this work is to correlate these lipid molecules to disease state and progression. This is the first reported study of its kind on these tissues.
\end{abstract}

\section{Introduction}

Chronic otomastoiditis refers to an inflammatory condition involving the middle ear and mastoid compartments. In the
United States alone, chronic otomastoiditis is estimated to account for over 30 million physician visits each year with an annual cost of roughly 3 to 5 billion dollars, and it remains a common indication for antibiotic therapy and surgery. ${ }^{1}$ received

September 25, 2014

accepted

November 6, 2014

published online

December 8, 2014
DOI http://dx.doi.org/

10.1055/s-0034-1396522.

ISSN 1809-9777.
Copyright (c) 2015 by Thieme Publicações License terms

Ltda, Rio de Janeiro, Brazil 
Interestingly, this pathology is also found in a significant proportion of indigenous children from the United States, Canada, Northern Europe, Australia, and New Zealand, the implication of which is obscure. ${ }^{2}$ Chronic otomastoiditis can result in hearing loss, vertigo, facial paralysis, sigmoid sinus thrombosis, meningitis, increased intracranial pressure, brain abscess, and in rare cases can even lead to death. In this condition, the normally air-filled middle ear and mastoid compartments become infiltrated and opacified by soft tissue-like material, which frequently requires surgical removal because resolution of symptoms is not achieved by antibiotic, anti-inflammatory, or other treatments.

When the surgically removed mastoid tissue is evaluated by histologic methods, chronic inflammation is almost always seen as demonstrated by an infiltration of polymorphonuclear cells and lymphocytes as well as a deposition of dense fibrotic tissue. Interestingly, standard swab and plate culture techniques rarely identify any associated pathogens. Several possibilities have been proposed to account for the production of this material, including infectious, autoimmune, neoplastic, and allergic processes. Despite the negative results with swab and plate techniques, an infectious etiology should not be excluded as there are plausible explanations as to why pathogens have evaded detection. These include the presence of biofilms, polysaccharide matrices created by bacteria that protect or shield them from the host response. Once enveloped within the biofilm, bacteria are difficult to detect by standard swab and plate techniques. The true relationship between mastoiditis and this material is still unknown. This continues to be a significant barrier toward clinical progress for treatment.

Lipids play crucial roles in cell, tissue, and organ physiology; not only do they provide an energy reservoir and serve as structural components of membranes, but they also act as signaling molecules and hormone precursors. Consequently, their steady-state concentrations are under tight homeostatic control, ${ }^{3,4}$ perturbation of which leads to disease and injury. Lipidomics aims to profile lipids such that the data sets can be used to compare control and disease states. Relating the lipidome to the proteome and the genome is an emerging field that provides an experimental platform with which complex biochemical systems can be related to genetic, developmental, and environmental influences; superimposed over these factors, the effect of disease states can be studied. With the ultimate goal of identifying lipid biomarkers of otomastoiditis, the first step is to identify the major lipid components of the involved tissues. In this report, we used high-performance thin-layer chromatography (HPTLC), combined gas chromatography (GC)/electron impact (EI)mass spectrometry (MS), and flow-injection (FI)/electrospray ionization (ESI)-tandem mass spectrometry (MSMS) to qualitatively identify the major lipid classes in the mastoid tissue and mastoid compartment bone dust (BD). A necessary first step toward elucidating the composition and mechanisms behind its production, this information is fundamental to further identification of clinical biomarkers for otomastoiditis, and subsequently efficient patient diagnosis and treatment.

\section{Methods}

\section{Solvents}

Chloroform, methanol, and formic acid (FA) were purchased from Fisher Scientific (San Jose, California, United States). Triethylamine (TEA), butylated hydroxytoluene (BHT), and ammonium acetate (AA) were purchased from Sigma Aldrich (St. Louis, Missouri, United States).

\section{Patients Recruitment and Biological Sample Collection}

With approval from and strict adherence to the Medical Institutional Review Board guidelines and regulations, samples were collected after written consent was obtained before surgery from patients with and without chronic otomastoiditis.

Recruited patients included those who showed clinical symptoms consistent with the diagnosis such as otorrhea, hearing loss, and otalgia. Furthermore, these patients showed the presence of pathologic mastoid tissue, as revealed by a computed tomography scan. Four additional cases of patients undergoing mastoidectomy during exposure of the facial nerve for parotid tumors were recruited to participate in this study and provide samples for the BD group.

\section{Sample Collection}

In the operating room, mastoid tissue was excised into sterile plastic specimen cups, and BD was collected after drilling through the mastoid bone. Specimens were stored on ice for transportation to the laboratory, where they were then transferred to $1.5-\mathrm{mL}$ microcentrifuge tubes, to which 500 $\mu \mathrm{L}$ of ice-cold deionized water was added. Tubes were mixed briefly to wash and remove excess blood, then centrifuged at low speed $(2,000 \mathrm{~g})$. The supernatant was discarded and the process was repeated twice. Samples were then stored at $-80^{\circ} \mathrm{C}$.

\section{Sample Extraction}

To minimize hydrolysis or degradation, the workup was done on ice as swiftly as possible. Samples were thawed at room temperature and then placed on ice. A small piece of chronic otomastoiditis tissue (COT) from each patient was placed on an ethanol-cleaned watch glass, also on ice, and diced (roughly $500-\mu \mathrm{m}^{2}$ pieces) with a no. 10 scalpel (sterilized for 30 minutes in ethanol) to aid in homogenization. The BD was already amenable to homogenization and extraction, so this consideration was unnecessary. To prevent oxidation, BHT (final concentration $300 \mu \mathrm{M}$ ) was added to all solutions and solvents used throughout the lipid extraction procedures. Roughly $1 \mathrm{mg}$ of tissue from each patient's COT was pooled into a glass test tube and roughly $20 \mathrm{mg}$ of BD from each patient were pooled into another tube, and $1 \mathrm{~mL}$ of deionized water containing BHT $(\mathrm{dH} 2 \mathrm{O}+\mathrm{BHT})$ was added to both. The pooled samples were then homogenized while on ice, either with an ultra-sonic cell disrupter (Branson, three 15-second bursts with a 5-minute interval in between) or a Brinkman Polytron Homogenizer (30 seconds).

After settling, $10 \mu \mathrm{L}$ aliquots of the supernatants were diluted 1:100 in $\mathrm{dH} 2 \mathrm{O}+\mathrm{BHT}$ and the optical density 
determined at $417 \mathrm{~nm}$ against a water blank (Beckman DU 640B spectrophotomer). ${ }^{5}$ A $60-\mu \mathrm{L}$ aliquot of freshly drawn human blood was diluted in $1 \mathrm{~mL} \mathrm{dH} 2 \mathrm{O}+\mathrm{BHT}$, sonicated as described above, and the OD417nm determined. From a comparison of the absorption intensities, control human blood samples were then prepared at an appropriate dilution in $\mathrm{dH} 2 \mathrm{O}+\mathrm{BHT}$ to match the hemoglobin, and theoretically, the blood content of each tissue sample.

The remainder of each homogenized suspension and the matched human blood samples (990 $\mu \mathrm{L}$ each) were then subjected to a modified Bligh-Dyer lipid extraction. ${ }^{6,7}$ In an aqueous suspension, $1,237.5 \mu \mathrm{L}$ of chloroform containing 300 $\mu \mathrm{M}$ BHT $(\mathrm{CHCl} 3+\mathrm{BHT})$ and 2,475 $\mu \mathrm{L}$ methanol containing $300 \mu \mathrm{M}$ BHT $(\mathrm{MeOH}+\mathrm{BHT})$ were added to each sample to achieve a chloroform:methanol:water ratio of $1: 2: 0.8, \mathrm{v} / \mathrm{v} / \mathrm{v}$. Samples were mixed thoroughly, and after 30 minutes at $27^{\circ}$ C, additional $\mathrm{CHCl} 3+\mathrm{BHT}(1,237.5 \mu \mathrm{L})$ and $\mathrm{dH} 2 \mathrm{O}+\mathrm{BHT}$ $(1,237.5 \mu \mathrm{L})$ were added to give the final chloroform:methanol:water ratio of $1: 1: 0.9, \mathrm{v} / \mathrm{v} / \mathrm{v}$. The samples were mixed thoroughly again, centrifuged $(2,000 \mathrm{~g}$ for 3 minutes), and the chloroform (bottom) phase was transferred with a glass syringe to $15-\mathrm{mL}$ glass capped test tubes. The extraction was repeated once using a volume of $\mathrm{CHCl} 3+\mathrm{BHT}$ identical to that removed in the previous step. The pooled chloroform phases were dried under a gentle stream of dry nitrogen gas, capped, and stored at $-20^{\circ} \mathrm{C}$.

\section{High-Performance Thin-Layer Chromatography}

HPTLC was performed to elucidate the complexity of the tissue samples and broadly identify major lipid classes. ${ }^{7}$ Each sample was redissolved in $100 \mu \mathrm{L}$ of chloroform, from which a $5-\mu \mathrm{L}$ aliquot was loaded as a $0.8-\mathrm{cm}$ band onto a prewashed and

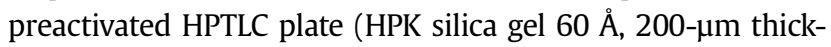
ness, $10 \times 10 \mathrm{~cm}$, Whatman, Kent, United Kingdom), prewashed in chloroform-methanol 1:1 (v/v), dried, and immediately prior to use heat-activated at $100^{\circ} \mathrm{C}$ for 1 hour, ${ }^{7}$ along with lipid standards as a separate band (lactosylceramide [CerLac, $2 \mu \mathrm{g}$; Sigma Aldrich], triacylglycerol [TAG, $5 \mu \mathrm{g}$; Sigma Aldrich], phosphatidylcholine [PtdCho; $5 \mu \mathrm{g}$; Avanti Polar Lipids, Alabaster, AL], cholesterol [C, $5 \mu \mathrm{g}$; Avanti Polar Lipids], and sphingomyelin [CerPCho, $5 \mu \mathrm{g}$; Sigma Aldrich]). After developing in chloroform/methanol/water (65:25:4, v/v/v), the plate was sprayed with orcinol $(0.02 \%$ in $50 \%$ H2SO4; Sigma Aldrich) and heated $\left(120^{\circ} \mathrm{C}, 2\right.$ to 3 minutes). An image of the stained plate was recorded digitally. The plate was then further stained with Coomassie Brilliant Blue G-250 (0.03\% in 30\% methanol/100 mM NaCl; Biorad, Hercules, CA) and destained with methanol (30\% in $100 \mathrm{mM} \mathrm{NaCl}){ }^{8,9}$ Another image of the plate was recorded digitally.

The remaining $90 \mu \mathrm{L}$ of each sample was loaded as an $8-\mathrm{cm}$ band onto a longitudinally prescored HPTLC plate and developed as described above. A narrow strip of this plate (5\%) was detached (along the score) and stained with orcinol as described above. Based on the orcinol staining pattern the unstained part was divided into four zones (zone 1, relative to front (Rf) 0.0 to 0.24 ; zone 2 Rf 0.24 to 0.47 ; zone 3 Rf 0.47 to 0.71 ; zone 4 Rf 0.71 to 1.0 ), and the silica from each was scraped into a clean glass tube (one zone/tube). The scrapings were treated with chloroform/methanol ( $1 \mathrm{~mL}, 1: 1, \mathrm{v} / \mathrm{v})$, and after centrifugation $(2,000 \mathrm{~g}, 5$ minutes $)$ the supernatants were collected into a new glass tube and the silica pellet was re-extracted once. The pooled supernatants were subjected to a Bligh-Dyer extraction by the addition of water $(0.9 \mathrm{~mL})$, the phases separated by centrifugation $(2,000 \mathrm{~g}$, 5 minutes) and the chloroform phases were removed to clean glass vials. The Bligh-Dyer extraction was repeated once and the pooled chloroform phases were dried under a stream of nitrogen gas, resulting in four HPTLC fractions per sample. The dried samples were stored at $-20^{\circ} \mathrm{C}$.

\section{Gas Chromatography/Mass Spectrometry of the High- Performance Thin-Layer Chromatography Zones}

Each lipid fraction extracted from the HPTLC plate was redissolved in chloroform:methanol $(500 \mu \mathrm{L}, 1: 1)$. Fifty microliters of each sample were transferred to glass conical GC injector vials and dried under a nitrogen stream. Each sample was then treated with $50 \mu \mathrm{L} \mathrm{N}, \mathrm{O}$-bis(trimethylsilyl)trifluoroacetamide containing $10 \%(\mathrm{v} / \mathrm{v})$ trimethylchlorosilane and incubated $\left(60^{\circ} \mathrm{C}, 60\right.$ minute) to convert all carboxyl, amino, and hydroxyl functional groups to their corresponding trimethylsilyl derivatives. ${ }^{10,11}$ The derivatized samples were then placed in an autosampler (Agilent 7683 series, Foster City, California, United States) from which an aliquot of each ( $1 \mu \mathrm{L}$, equivalent of $0.2 \%$ of the contents of the HPTLC fraction) was injected onto a bonded-phase nonpolar fused silica capillary column (Phenomenex ZB-5, phenyl/dimethylpolysiloxane 5/95, $60 \mathrm{~m} \times 0.25 \mathrm{~mm}, 0.10-\mu \mathrm{m}$ film thickness, Phenomenex, Torrance, California, United States; injector port $250^{\circ} \mathrm{C}$ ) and eluted (constant flow, $1 \mathrm{~mL} / \mathrm{min}$ ) with ultra-high-purity helium (Agilent 6890A GC system) over a 63-minute temperature $\operatorname{ramp}\left(\min /{ }^{\circ} \mathrm{C}: 0 / 50,3 / 50,53 / 300,63 /\right.$ $300)$. The end of the column (GC/MS transfer line at $250^{\circ} \mathrm{C}$ ) was directly inserted into the EI source $\left(180^{\circ} \mathrm{C}, 70 \mathrm{eV}\right)$ of a time-of-flight mass spectrometer (Waters GCT, calibrated with perfluorotributylamine immediately prior to the analysis of each batch of samples) scanning from $\mathrm{m} / \mathrm{z} 40$ to 650 ( $0.9 \mathrm{~s} / \mathrm{scan}$ at a resolution [full width at half maximum] of 7,000 , lock-mass from column bleed at 207.0329 C5H15Si303) with a 15-minute solvent delay. Data were collected with instrument manufacturer-supplied software (MassLynx version 4.1, Waters Corporation, Milford, MA). Each sample was individually prepared and run in duplicate.

Identifications were based on comparison of spectra averaged over the width of the GC peaks within the total ion chromatogram, with background subtraction, to the National Institute of Standards and Technology (NIST) 2008 Mass Spectral Library (version 2.0f). These were based on NIST match factors of at least 750 , indicating strong concordance between the unknowns and the library spectra, and acceptable visual concordance between the unknown and library spectra.

\section{Electrospray Ionization/Tandem Mass Spectrometry}

To screen for the major lipid classes on the basis of tandem mass spectra, identical lipid extracts were prepared as described above and dissolved in chloroform:methanol $(500 \mu \mathrm{L}$, 1:1). To provide the appropriate milieu for effective 
ionization, a $50-\mu \mathrm{L}$ aliquot of each stock solution was diluted $1: 5(\mathrm{v} / \mathrm{v})$ in chloroform:methanol:formic acid $(200 \mu \mathrm{L}$, $50: 50: 0.1, v / v / v)$. Another $50 \mu \mathrm{L}$ of each stock was diluted similarly in chloroform:methanol:triethylamine $(200 \mu \mathrm{L}$, $50: 50: 0.1, v / v / v)$, and a third $50-\mu \mathrm{L}$ aliquot was diluted similarly in chloroform:methanol:ammonium acetate (200 $\mu \mathrm{L}, 50: 50: 10 \mathrm{mM}, \mathrm{v} / \mathrm{v} / \mathrm{c}$ ). Ten microliters of each of these dilutions, from each sample, were analyzed on an Agilent 6460 triple quadrupole mass spectrometer by flow injection into an Agilent Jet Stream ion source (gas temperature $300^{\circ} \mathrm{C}$, gas flow $6 \mathrm{~L} / \mathrm{min}$, nebulizer pressure $45 \mathrm{psi}$, sheath gas temperature $50^{\circ} \mathrm{C}$, sheath gas flow rate $10 \mathrm{~L} / \mathrm{min}$, capillary voltage $4,500 \mathrm{~V}$, nozzle voltage $2,000 \mathrm{~V}$ ) with chloroform: methanol (50:50) as the running solvent $(100 \%, 0.050 \mathrm{~mL} /$ min). Data were collected with instrument manufacturersupplied software (Agilent Mass Hunter version B.05.00).

Using published methods as a guide, ${ }^{12-21}$ optimal conditions for precursor, product, and neutral loss ion scans for the PtdCho/CerPCho, cholesteryl esters (CE), and sulfated lipids (S) were developed in-house using authentic standards (-Table 1). Optimization for each compound class was performed with solutions prepared in chloroform:methanol (50:50, v/v), $1 \mathrm{pmol} / \mu \mathrm{L}$ using $10-\mu \mathrm{L}$ injections, containing either $10 \mathrm{mM}$ AA (CE), 0.1\% TEA (phosphatidyl serine [PtdSer], phosphatidyl inositol [PtdIns], and S), or $0.1 \%$ FA (PtdCho/CerPCho and phosphatidyl ethanolamine [PtdEtn]). ${ }^{12}$ The standards utilized for this purpose were: PtdCho, diheptanoyl phosphatidylcholine and 1-heptadecanoyl-1-lyso-phosphatidylcholine (Sigma Aldrich); PtdIns, L- $\alpha$-phosphatidylinositol sodium salt from Glycine max (soybean; Sigma Aldrich); PtdSer, 3-sn-phosphatidyl-L-serine sodium salt from bovine brain (Sigma Aldrich); Cer, ceramide from bovine spinal cord (Sigma Aldrich); PtdEtn, 3-snphosphatidylethanolamine from bovine brain (Sigma Aldrich). Instrument parameters (collision energy, fragmentor, and cell accelerator voltage) were manually tuned with repeat injections until maximal signal intensity for precursors ions were obtained in each scan modality. Limits of detection for these compounds were then determined by preparing serial dilutions of the standard solutions and running them at the aforementioned optimized conditions until the dilution at which a signal 3 times as intense as background was determined. Optimized instrument parameters and limits of detection for CE and S were previously determined on this instrument via similar means. ${ }^{22-24}$

\section{Results}

\section{High-Performance Thin-Layer Chromatography Profiles}

HPTLC was used to estimate the magnitude of blood contamination in the extracts. The OD417nm provided a convenient method for measuring hemoglobin, and therefore blood that inevitably comprises a portion of each sample. The contribution of blood to the lipid profiles of the tissue samples was insignificant (-Fig. 1). Co-spotted matched blood control samples for each tissue sample revealed relatively minor bands with the exception of $C$ and possibly TAG, although the tissue samples were vastly stronger for these bands as well.

HPTLC was also used to gauge the complexity of the lipid profile in the samples and to screen for possible major differences between samples. Visual inspection of the plates revealed multiple bands in the region of PtdCho, CerPCho, bands in the region of CerLac, relatively minor bands between CerLac and $\mathrm{C}$, and strong bands at the Rf of $\mathrm{C}$ and TAG (-Fig. 1). The results suggested that the samples were not sufficiently complex to warrant prefractionation ahead of tandem MS.

\section{Gas Chromatography/Electron Impact-Mass Spectrometry Profiles of High-Performance Thin-Layer Chromatography Zones}

The total ion chromatograms revealed a peak for $\mathrm{C}$ in zone 4 extracts from each of the sample groups (-Fig. 2), which was overloaded in the COT sample, and on-scale for the BD sample. In addition, less intense peaks were identified in both zone 4 extracts for palmitic, oleic, and stearic acids. Glycerol and

Table 1 Scan modalities for tandem mass spectrometric screens of major lipid classes

\begin{tabular}{|l|l|l|l|l|l|l|l|}
\hline Lipid class & $\begin{array}{l}\text { Dilution } \\
\text { used }\end{array}$ & $\begin{array}{l}\text { lons or } \\
\text { neutral mass }\end{array}$ & Polarity & $\begin{array}{l}\text { Type of } \\
\text { scan }\end{array}$ & Fragmentor & $\begin{array}{l}\text { Collision } \\
\text { energy }\end{array}$ & $\begin{array}{l}\text { LOD } \\
\text { (injected in } \mathbf{1 0} \mu \mathrm{L})\end{array}$ \\
\hline PtdCho/CerPCho & FA & $\mathrm{m} / \mathrm{z} 184$ & Positive & Precursor & 100 & 20 & $450 \mathrm{fmol}$ \\
\hline PtdSer & TEA & 87 & Negative & Neutral loss & 45 & 21 & $40 \mathrm{pmol}$ \\
\hline PtdEtn & FA & 141 & Positive & Neutral loss & 141 & 10 & $5 \mathrm{pmol}$ \\
\hline PtdIns & TEA & $\mathrm{m} / \mathrm{z} 241$ & Negative & Precursor & 200 & 50 & $50 \mathrm{pmol}$ \\
\hline Cer & FA & $\mathrm{m} / \mathrm{z} 264.1$ & Positive & Precursor & 150 & 30 & $100 \mathrm{pmol}$ \\
\hline CE & And 265.3 & $\mathrm{m} / \mathrm{z} 369.4$ & Positive & Precursor & 80 & 7 & - \\
\hline S & TEA & $\mathrm{m} / \mathrm{z} 97$ & Negative & Precursor & 190 & 85 & $200 \mathrm{fmol}$ \\
\hline
\end{tabular}

Abbreviations: AA, ammonium acetate; CE, cholesteryl esters; Cer, ceramide; CerPCho, sphingomyelin; FA, formic acid; LOD, limit of detection; PtdCho, phosphatidyl choline; PtdSer, phosphatidyl serine; PtdEtn, phosphatidyl ethanolamine; Ptdlns, phosphatidyl inositol; S, sulfated lipids; TEA, triethyl ammonia.

Notes: LOD for CE not found due to high background at low concentrations. The LOD was determined from serial dilutions of standards prepared inhouse, and the assigned value was the amount injected that gave a signal $\sim 3$ times more intense than background. 


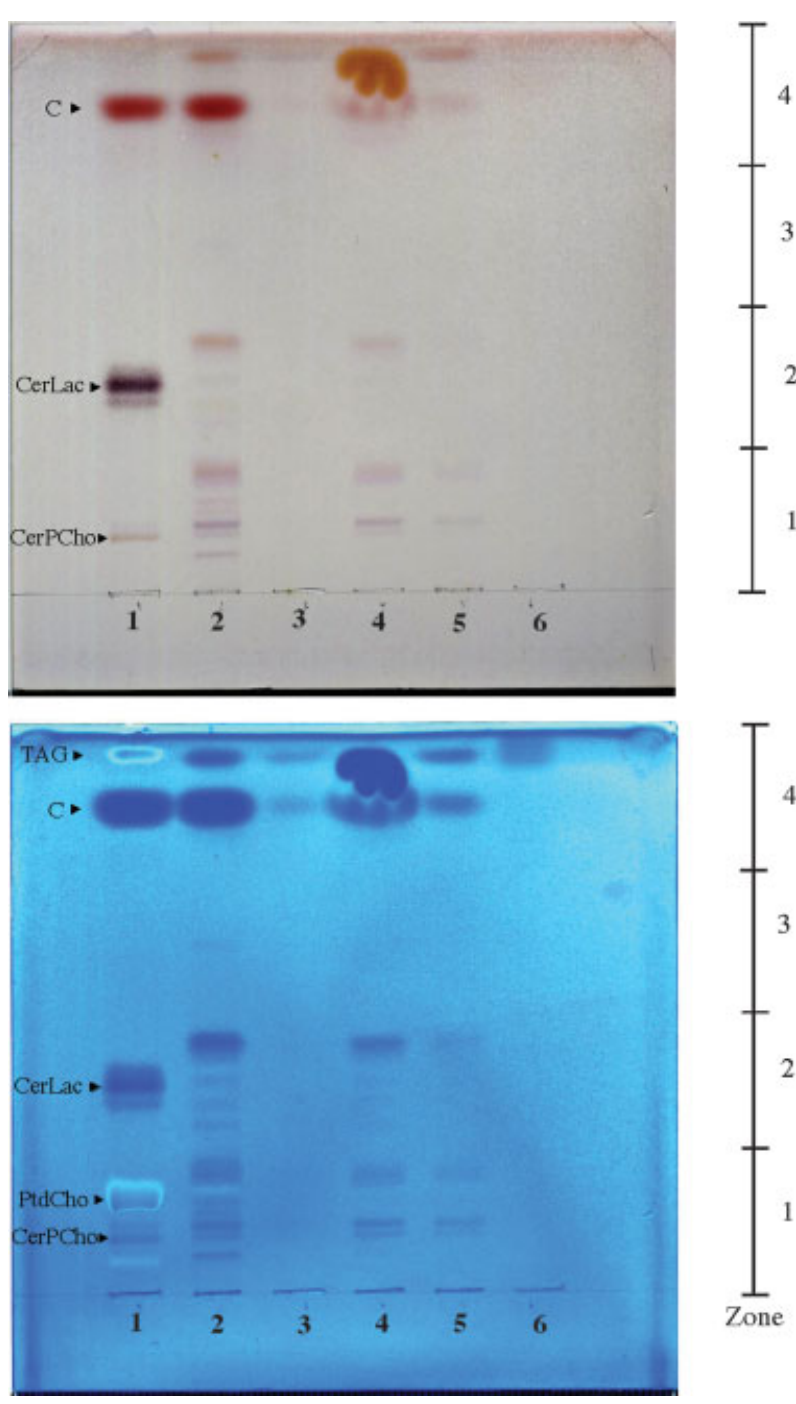

Fig. 1 Orcinol-stained (top panel) and Coomassie blue-stained (bottom panel) high-performance thin-layer chromatography (HPTLC) displays of (left to right): 1 , standards ( $5 \mu \mathrm{g} /$ compound except CerLac 2 $\mu \mathrm{g}) ; 2$, mastoid tissue; 4 , bone dust; 6 , blank. Matched blood controls for each sample are in the adjoining lanes (for example, for lane 2 the matched blood control is in lane 3). The plates were run and first stained with orcinol and then Coomassie as described in the Methods. The HPTLC-purified samples that were separately prepared for mass spectrometric analysis were: zone 1 , Rf 0.0 to 0.24 ; zone 2 Rf 0.24 to 0.47 ; zone $3 \mathrm{Rf} 0.47$ to 0.71 ; zone $4 \mathrm{Rf} 0.71$ to 1.0 . Abbreviations: C, cholesterol; CerLac, lactosylceramide; CerPCho, sphingomyelin; TAG, triacylglycerol; PtdCho, phosphatidylcholine.

phosphate were also identified; however, their presence in all four zones of both COT and BD relegated them to the status of contaminants. Based on an estimated limit of detection of $1 \mathrm{ng}$ injected, the inferred content of any free fatty acids in the samples must be greater than $\sim 5.5 \mathrm{ng}$ per $\mathrm{mg}$ of COT and 260 pg per mg of BD.

\section{Flow-Injection/Electrospray Ionization-Tandem Mass Spectrometry Profiles}

The MSMS approach to identify the components of the major lipid classes using flow injection with appropriately selected

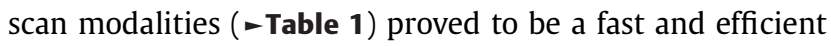

screen. Using the redissolved Bligh/Dyer extracts, there was no evidence of ion suppression as learned from repeat injections of 10 -fold concentrated samples from which there was the expected increase in signal intensity. The lack of ion suppression observed during flow injection validated the decision to forgo chromatography. Consequently, the analyses were both faster and shorter, adding to the efficiency of this screening method. The criteria for selection of significant ions from these spectra were that the same signal must be present in repeat injections from a given sample, and the peak intensity must be at least fivefold larger than the local baseline. Such stringent criteria were used to restrict this report to the most abundant species and keep the number of assigned peaks to a manageable level. The estimated limits of detection (LOD) for the different lipid classes varied from 200 and $450 \mathrm{fmol}$, to 5, 40, 50, and 100 pmol injected from $\mathrm{S}$, PC/SM, PE, PS, PI, and C, respectively (-Table 1). These injected LODs were determined by injection of serial dilutions of the authentic standards as discussed above, and equate to tissue LODs between $125 \mathrm{fmol} / \mathrm{mg}(\mathrm{S})$ and $62.5 \mathrm{pmol} / \mathrm{mg}$ (Cer). Ion assignments in the collected spectra were made as protonated $\left(\mathrm{MH}^{+}\right)$and ammoniated $\left(\mathrm{M}+\mathrm{NH} 4^{+}\right)$molecules when FA and AA solvents were used, respectively.

The complexity of the spectra varied for the different classes, and representative MSMS spectra for the majorly present lipid classes (-Fig. 3) show the most abundant species in each. - Tables 2, 3, and $\mathbf{4}$ show precursor ions for four major lipid classes identified (PtdCho, PtdEtn, Cer, and $\mathrm{CE}$, respectively) and the identity of the most intense ion from each sample. Signals for PtdIns, PtdSer, and S were not detected in any sample.

The parents of $\mathrm{m} / \mathrm{z} 184$ positive ion scan modality, used to screen for choline-containing lipids, relies on the facile production of the $\mathrm{C} 5 \mathrm{H} 15 \mathrm{PO} 4 \mathrm{~N}^{+}$fragment ion produced during collisionally activated dissociation (CAD) from all PtdChos by cleavage of the moiety attached to the sn3 position of the glycerol backbone. ${ }^{25}$ Even with stringent selection criteria, in FA, this scan modality revealed a complex array of signals in the COT sample with more than 20 species present (-Fig. 3, - Table 2, even-numbered $\mathrm{m} / \mathrm{z}$ values). However, the same scan and selection criteria in FA solvent revealed only two signals in the BD sample. The pair of ions at $\mathrm{m} / \mathrm{z} 758$ and 760 , presumably differing by a single double bond, dominated both profiles and are assigned as 34a:2 and 34a:1 diacyl phosphatidylcholine, respectively. When identical samples were diluted in AA solvent, the profile from COT was even more complex, and astonishingly the profile from BD was equally complex with 24 and 20 assigned signals (even-numbered $\mathrm{m} / \mathrm{z}$ values), respectively. Sprinkled among the even $\mathrm{m} / \mathrm{z}$ signals are a small number of odd mass assignments.

The neutral loss of 141 Da positive ion scan mode was used to profile PtdEtns. ${ }^{21}$ This was based on the facile loss of the C2H804NP molecule from the protonated PtdEtn molecular ions during CAD. COT revealed up to 8 and 14 different species in FA- and AA-containing solvent, respectively (-Fig. 3, - Table 3). BD revealed two and nine different species in the same solvents, respectively. The most abundant 

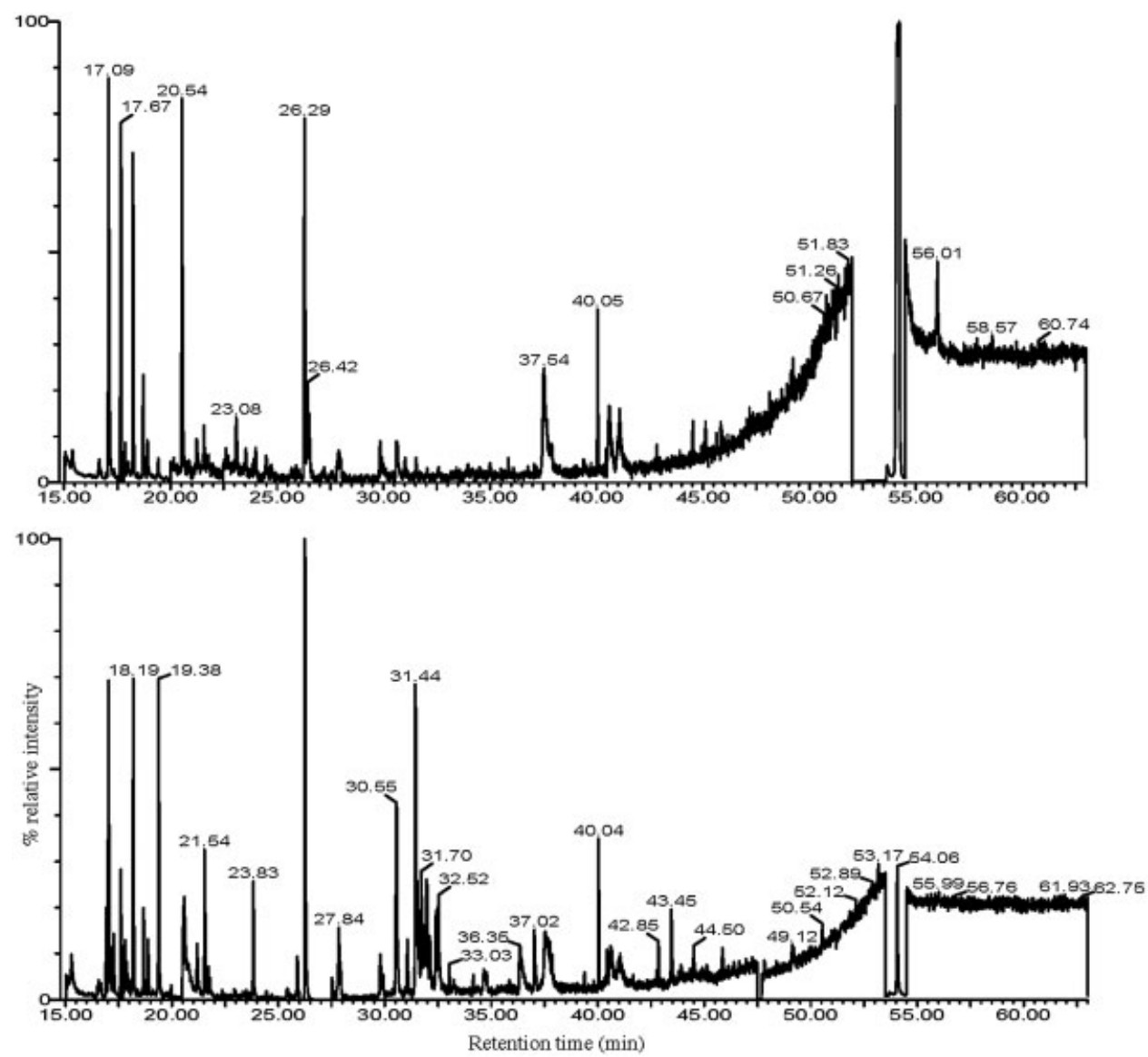

Fig. 2 Combined gas chromatography/electron impact-mass spectrometry total ion current chromatograms from high-performance thin-layer chromatography (HPTLC) zone 4 extracts. Total ion current chromatograms of HPTLC zone 4 trimethylsilyl (TMS)-derivatized eluates from bone dust (top panel) collected during mastoidectomy and tissue excised from the mastoid cavity (bottom panel) in patients with chronic otomastoiditis. The dominant peak in each chromatogram (retention time 54.06 minutes) was identified as cholesterol. Other tentative identifications include: glycerol (20.54 minutes), palmitic acid (37.02 minutes), oleic acid (40.59 minutes), and stearic acid (41.04 minutes). The region from 15.00 to 20.00 minutes is populated by byproducts of the derivatization reaction and excess derivatizing reagents, and the large peak at 26.25 minutes in the bottom panel was identified at butylated hydroxytoluene. To aid in visualizing smaller peaks, the ordinate of each chromatogram was regionally expanded as follows: top panel: 15.03 to $17.71(\times 10), 17.79$ to $19.89(\times 24), 19.99$ to $52.00(\times 186), 54.50$ to 63.00 ( $\times 54)$; bottom panel: 15.03 to 17.51 ( $\times 16), 17.69$ to $25.99(\times 36), 27.49$ to $47.50(\times 54), 47.70$ to $53.50(\times 36), 54.50$ to $63.00(\times 24)$. The fatty acids observed in these chromatograms were present in abundance below the limit of confident identification, thus the spectra were not of high quality.

species in all samples were the pair of ions at $\mathrm{m} / \mathrm{z} 768$ and 766 , corresponding to 38a:4 and 38a:5 diacyl phosphatidylethanolamine, respectively. The profiles also revealed a few odd mass assignments.

The parents of $\mathrm{m} / \mathrm{z} 369$ positive ion scan modality in AAcontaining solvent was used to profile CEs. ${ }^{24}$ This scan modality was based on the facile fragmentation of the all $\mathrm{CE}\left(\mathrm{M}+\mathrm{NH}_{4}\right)^{+}$parents yielding an intense ion for the cholesterol-containing fragment $(\mathrm{C} 27 \mathrm{H} 49)$ during CAD. COT and $\mathrm{BD}$ revealed 22 and 2 different molecular species, respectively, of which the signal at $\mathrm{m} / \mathrm{z} 666$ corresponding to the linoleate ester was most intense in both (-Fig. 3, - Table 4).

The scan modality for PtdSer, the neutral loss of $87 \mathrm{Da}$ in the negative ion mode in TEA solvent, was based on the loss of the serine functionality $\left(\mathrm{C} 3 \mathrm{H} 5 \mathrm{NO}_{2}\right)$ from the $(\mathrm{M}-\mathrm{H})^{-}$parent ion common to these compounds. ${ }^{21}$ The scan modality for PtdIns was based on the parents of the common fragment ion at $\mathrm{m} / \mathrm{z} 241$ (C6H10O8P) in the negative ion mode in TEA solvent formed in abundance under CAD conditions with these compounds. ${ }^{26}$ The scan modality for Cer, parents of $\mathrm{m} / \mathrm{z}$ 264, was based on the formation of a common positive rearrangement CAD fragment ion $\mathrm{C} 18 \mathrm{H} 34 \mathrm{~N}$ displayed by these compounds in FA solvent. ${ }^{27}$ The scan modality for $\mathrm{S}$ was based on extensive in-house experience with these lipids ${ }^{28,29}$ in which the common negative fragment ion at $\mathrm{m} / \mathrm{z} 97\left(\mathrm{HSO}^{-}\right.$) forms the basis of the scan in the negative ion mode in TEA solvent. Despite careful optimization of scan parameters for each of these modalities, using authentic standards, signals for PtdIns, PtdSer, Cer, and S from COT and $\mathrm{BD}$ were below their respective limits of detection ( - Table 1).

\section{Discussion}

There is no one technique capable of conveniently displaying all lipids in a sample. As such, we have used a combination of 

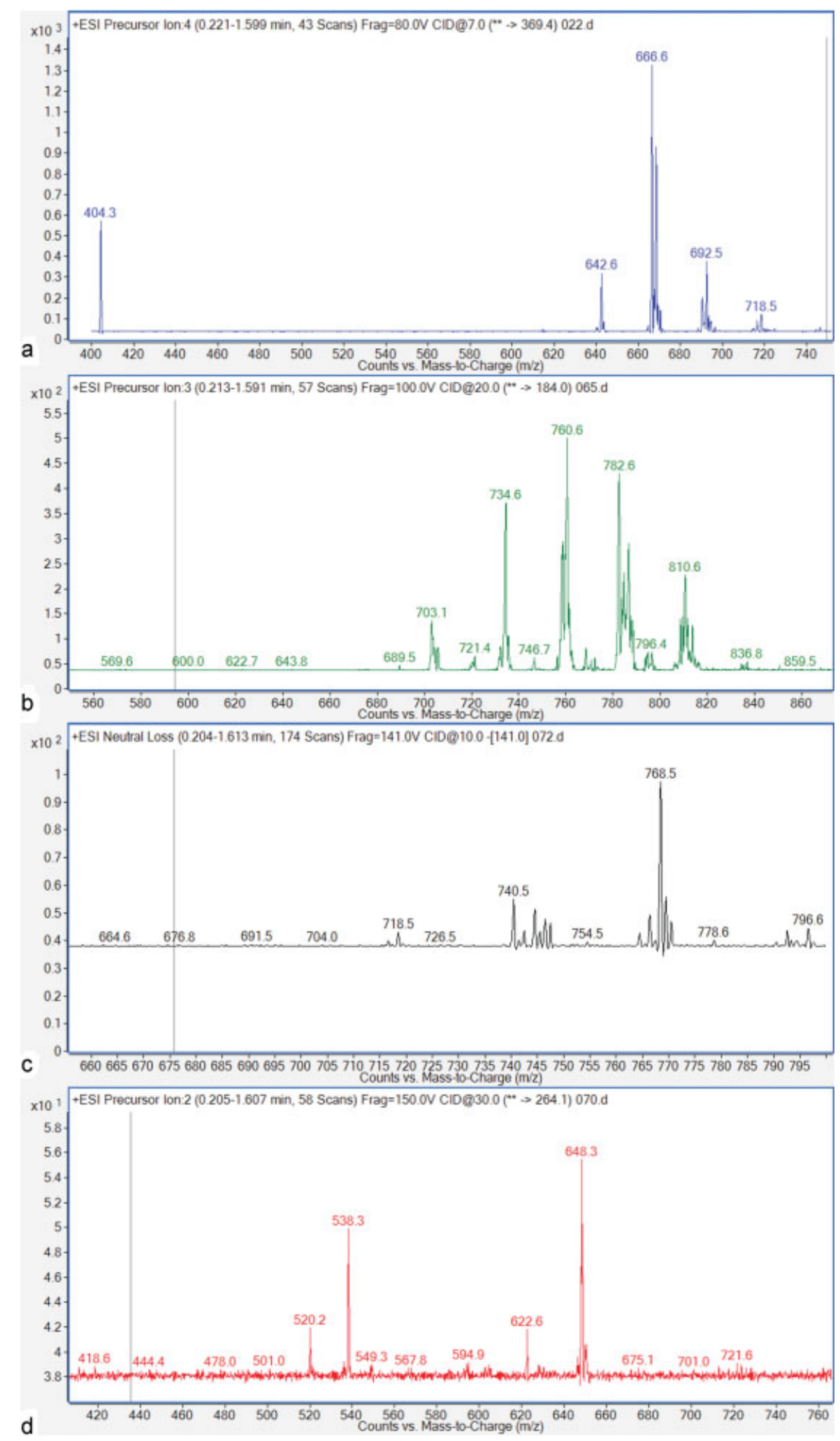

Fig. 3 Representative tandem mass spectrometry spectra of the majorly present lipid classes in mastoid tissue. (a) Precursor ion scan of m/z 369.4 shows cholesteryl esters and similar compounds. (b) Precursor ion scan of $\mathrm{m} / \mathrm{z} 184$ of samples shows phosphatidylcholines, sphingomyelins, and similar compounds. (c) Neutral loss scan of $\mathrm{m} / \mathrm{z} 141$ of samples shows phosphatidylethanolamines and similar compounds. (d) Precursor ion scan of $\mathrm{m} / \mathrm{z} 264.1$ shows ceramides and similar compounds. Compounds not shown here were present below the limit of detection. 
Table 2 Tabulation of the major ions evident in the precursor of $\mathrm{m} / \mathrm{z} 184$ scan modality, characteristic of phosphatidylcholinecontaining species

\begin{tabular}{|c|c|c|c|}
\hline Tissue type & $\begin{array}{l}\text { Ion-pair } \\
\text { reagent }\end{array}$ & lons $^{a}$ & $\begin{array}{l}\text { Identification of most } \\
\text { intense species }\end{array}$ \\
\hline \multirow[t]{2}{*}{ COT } & FA & $\begin{array}{l}\text { 496.4, 544.4, 703.5, } 720.3,732.3,734.6,735.7,,^{b} \\
746.7,758.5,760.4,768.4,770.7,772.2,782.5, \\
784.6,786.6,794.4,796.4,808.6,810.5,834.4,836.8\end{array}$ & 34a:1 diacyl phosphatidylcholine \\
\hline & AA & $\begin{array}{l}\text { 520.4, 522.4, 544.1, 675.1, }{ }^{\mathrm{b}} 703.6,^{\mathrm{b}} 717.8,^{\mathrm{b}} 720.5, \\
732.7,734.5,744.8,746.6,748.5,758.6,760.6,768.4, \\
782.6,784.6,786.6,794.6,796.3,806.4,808.5,810.6 \\
812.7,832.6,834.4,836.5\end{array}$ & 34e:2 alkyl ether phosphatidylcholine \\
\hline \multirow[t]{2}{*}{ BD } & FA & $703.6,^{b} 758.4,760.6$ & 34a:2 diacyl phosphatidylcholine \\
\hline & AA & $\begin{array}{l}\text { 496.6, 520.4, 544, 675.1, }{ }^{\mathrm{b}} 676.3,689.6,^{\mathrm{b}} 703.5^{\mathrm{b}} 732.4, \\
734.3,744.1,746.6,748.3,758.5,760.5,768.3,782.5, \\
784.5,786.3,794.2,799.7, \mathrm{~b} 802.5,806.4,808, \\
810.4,813.8^{\mathrm{b}}\end{array}$ & Unknown \\
\hline
\end{tabular}

Abbreviations: AA, ammonium acetate; BD, bone dust; COT, chronic otomastoiditis tissue; FA, formic acid.

Notes: This listing includes only the signals that were more intense than fivefold above baseline. Close inspection of the spectra reveals many less intense signals. The LOD was determined from serial dilutions of standards prepared in-house, and the assigned value was the amount injected that gave a signal $\sim 3$ times more intense than background.

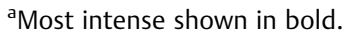

bodd-numbered $\mathrm{m} / \mathrm{z}$ values.

HPTLC, GC/EI-MS, and FI/ESI-MSMS to probe the lipidome of these pathologic tissues. The different techniques provide complementary and partially overlapping profiles. To our knowledge, this is the first study of its kind to evaluate the lipid characteristics of mastoid bone and tissue, and it is a vital first step toward elucidation of putative biomarkers for otomastoiditis. The HPTLC profiles of Bligh-Dyer extracted samples provide convenient visualization of the major lipid components in one display, with the single major limitation of an LOD of $\sim 0.5 \mu \mathrm{g} /$ compound ( $1 \mathrm{nmol}$ with a molecular weight of 500). HPTLC is therefore a convenient way to gauge the complexity of any sample, and is particularly useful in this case where there is no precedence for analysis of middle ear bone and tissues. In contrast, MS has unrivalled sensitivity and specificity with the single major limitation being the lack of a scan modality that can be applied to all compounds. Because this work was not sample limited, we have used a combination of HPTLC and MS to provide a global qualitative analysis of the lipid components of bone and tissue from the two patient classes.

Inclusion of a blood control for each tissue type on the HPTLC chromatogram showed a relatively sparse banding pattern, indicating an insignificant contribution of blood to the tissue extracts. A variable blood contamination is unavoidable when working with any tissue and bone samples excised from living patients.

The HPTLC profiles revealed a well-defined lipidome with significant quantities in the $\mathrm{C}$ - and choline-containing

Table 3 Tabulation of the major ions evident in the neutral loss of 141-Da scan modality, characteristic of phosphatidylethanolamine-containing species

\begin{tabular}{|c|c|c|c|}
\hline $\begin{array}{l}\text { Tissue } \\
\text { type }\end{array}$ & $\begin{array}{l}\text { Ion-pair } \\
\text { reagent }\end{array}$ & lons (most intense in bold font) & Identification of most intense species \\
\hline \multirow[t]{2}{*}{ COT } & FA & $\begin{array}{l}740.5,742.5,744.5,746.5,747.6,^{b} 764.5,766.2,768.5, \\
769.5, \text {, } 770.6\end{array}$ & 38a:4 diacyl phosphatidylethanolamine \\
\hline & AA & $\begin{array}{l}\text { 716.4, 718.5, 740.5, 742.4, 744.5, 746.5, 754.5, 764.5, } \\
766.4,768.4,769.5,{ }^{b} 770.5,792.5,793.5,{ }^{b} 794.5,796.6\end{array}$ & 38a:4 diacyl phosphatidylethanolamine \\
\hline \multirow[t]{2}{*}{$\mathrm{BD}$} & FA & $766.5,768.2$ & 38a:5 diacyl phosphatidylethanolamine \\
\hline & AA & $\begin{array}{l}\text { 716.5, 718.5, 740.5, 742.6, 744.5, 764.5, 766.5, 768.4, } \\
770.6\end{array}$ & 38a:4 diacyl phosphatidylethanolamine \\
\hline
\end{tabular}

Abbreviations: AA, ammonium acetate; BD, bone dust; COT, chronic otomastoiditis tissue; FA, formic acid.

Notes: This listing includes only the signals that were more intense than fivefold above baseline. Close inspection of the spectra reveals many less intense signals. The LOD was determined from serial dilutions of standards prepared in-house, and the assigned value was the amount injected that gave a signal $\sim 3$ times more intense than background.

${ }^{a}$ Most intense shown in bold.

${ }^{\mathrm{b}}$ Odd-numbered $\mathrm{m} / \mathrm{z}$ values. 
Table 4 Tabulation of the major ions evident in the precursor of m/z 369 scan modality, characteristic of cholesteryl esters

\begin{tabular}{|l|l|l|l|}
\hline $\begin{array}{l}\text { Tissue } \\
\text { type }\end{array}$ & $\begin{array}{l}\text { lon-pair } \\
\text { reagent }\end{array}$ & lons (most intense in bold font) & $\begin{array}{l}\text { Name of most } \\
\text { intense species }\end{array}$ \\
\hline COT & AA & $\begin{array}{l}404.3,614.6,640.1,642.6,664.6,666.6,668.6,670.6, \\
690.5,692.5,694.7,696.5,714.8,716.5,718.5,720.4,\end{array}$ & Cholesteryl linoleate \\
& & $722.7,744.6,746.5,748.8,750.6,778.5$ & \\
\hline BD & AA & $404.2,666.6$ & Cholesteryl linoleate \\
\hline
\end{tabular}

Abbreviations: AA, ammonium acetate; BD, bone dust; COT, Chronic otomastoiditis tissue.

Notes: This listing includes only the signals that were more intense than 5-fold above baseline. Close inspection of the spectra reveals many less intense signals. The limit of detection for this scan modality is listed in - Table $\mathbf{1}$.

regions. By visual inspection, and considering that the amount of BD used in these analyses was 20 times by wet weight that of the tissue, BD had a lower lipid concentration across the chromatogram as compared with COT; however, there was no gross difference between the chromatograms, other than the difference in opacity attributable to the relatively higher richness of lipid content in tissue. This means the major lipid components of these tissues are similar in type, but not in amount. It is unlikely that a small subpopulation of each sample pool could dominate the chromatogram because each sample in each pool is present in roughly equal quantities.

The GC/EI-MS lipidomic profiling of the trimethylsilyl-oxime derivatives of Bligh-Dyer extracts of COT and BD samples revealed a similar lipid complexity; however, BD was comparatively deficient in $\mathrm{C}$. This phenomenon can likely be attributed to the relatively higher concentration of $\mathrm{C}$ inherent to tissue as compared with bone. The low abundance of free fatty acids is of note. These compounds are important components of intermediary metabolism. Their low concentrations could be indicative of a possible acellular nature of the samples.

The MSMS work provided a more sensitive and directed profiling of the lipid content of these samples. The most abundant lipids detected were members of the PtdEtn, PtdCho, and CE classes. PtdChos are the principal phospholipids in human cell membranes and are a pulmonary surfactant. ${ }^{30}$ They are typically found on the exoplasmic side of the cell membrane and play a role in membrane-mediated signaling. PtdChos are also mainly structural components of cellular membranes, but also serve roles in intracellular signaling, differentiation, proliferation, and programmed cell death. As expected, a variety of PtdChos were detected in COTwith either FA or AA solvents (-Table $\mathbf{2}$ ). The unexpected result was the emergence of signals from $\mathrm{BD}$ in $\mathrm{AA}$ solvent that were not observed in FA solvent. This observation is of methodological significance relevant to future lipidomic screening experiments. There is considerable concordance between the signals observed for COT (either solvent) and BD (AA solvent). Application of the nitrogen rule to this data set means all quaternary nitrogen-containing species would be recorded at an evennumbered $\mathrm{m} / \mathrm{z}$. The relatively few odd-valued signals are unlikely to be choline-containing species, and unraveling their structures is beyond the scope of this work.

PtdEtns are found in all living cells and account for nearly a quarter of all phospholipids. ${ }^{31-33}$ In human physiology, they are typically found in neural tissue, serving roles in membrane fusion and cytokinesis, lipoprotein release in the liver, and, pathologically, prion propagation without the involvement of other proteins or nucleic acids. In bacteria, however, PtdEtns are the principal phospholipids; therefore, their relative abundance ( - Table 3 ) may be indicative of a bacterial presence despite the failure of the swab and plate culture experiments previously referred to. Application of the nitrogen rule to putative PtdEtns would mean the assigned parent ions would be even when protonated (in FA solvent) and odd when ammoniated (only in AA solvent). The presence of the same ion in both FA and AA solvents implies that at least some of the ions detected in AA solvent are protonated. The few odd-value assignments are not likely to be PtdEtns.

Interestingly, CEs are among the major contributors to local lipidome in mastoid tissue and bone and are present in much greater abundance and variety in mastoid tissue. As shown in - Table 4, both cholesteryl arachidonate and cholesteryl linoleate are present in mastoid tissue, but only cholesteryl linoleate is present both tissue types studied here. These findings could be potentially significant. According to Do et al, cholesteryl arachidonate and cholesteryl linoleate show microbicidal effects in vitro, including against Pseudomonas aeruginosa, ${ }^{24}$ a common human pathogen whose infection causes generalized inflammation and potentially sepsis. The differential presence of these antimicrobial lipids across the different tissue types analyzed and the significance of the variety of these lipids in the idiopathic mastoid tissue will be a topic of future study.

PtdSer, PtdIns, Cer, and S were present below the limit of detection in these samples ( $40 \mathrm{pmol}, 50 \mathrm{pmol}, 100 \mathrm{pmol}$, and 200 fmol, respectively, injected; - Table 1). This is an unexpected result as PtdIns and PtdSer are relatively common cellular membrane structural phospholipids and sulfated lipids play roles in cell-surface signaling. ${ }^{34,35}$ The significance of this finding is obscure at the moment. This is not to say that these lipid classes do not exist in mastoid tissue and/or mastoid bone, but rather their respective concentrations in these tissues is low, and a more sensitive targeted assay for them is required. The latter will be explored in future work.

\section{Conclusions}

These results represent a first step to characterize the lipid profile of patients with otomastoiditis and to identify 
potential biomarkers of the disease. As there is a dearth of literature on lipidomic profiling in bone, this report sought to develop an efficient and catholic analytical method for these tissues. Given the large and diverse role of lipids in tissue structure and the regulation of local homeostasis, the lipidome of the mastoid compartment is a logical place to begin looking for candidate biomarkers. The techniques developed in this study will be exploited in further analyses of individual patient samples in the search for relative abundance of these major species as well as a broader global lipidome comparison.

\section{References}

1 Rovers MM, Schilder AG, Zielhuis GA, Rosenfeld RM. Otitis media. Lancet 2004;363(9407):465-473

2 Morris PS, Richmond P, Lehmann D, Leach AJ, Gunasekera H, Coates HL. New horizons: otitis media research in Australia. Med J Aust 2009;191(9, Suppl):S73-S77

3 Ameer F, Scandiuzzi L, Hasnain S, Kalbacher H, Zaidi N. De novo lipogenesis in health and disease. Metabolism 2014;63(7):895-902

4 de Kroon AI, Rijken PJ, De Smet CH. Checks and balances in membrane phospholipid class and acyl chain homeostasis, the yeast perspective. Prog Lipid Res 2013;52(4):374-394

5 Luthra A, Denisov IG, Sligar SG. Spectroscopic features of cytochrome $\mathrm{P} 450$ reaction intermediates. Arch Biochem Biophys 2011; 507(1):26-35

6 Bligh EG, Dyer WJ. A rapid method of total lipid extraction and purification. Can J Biochem Physiol 1959;37(8):911-917

7 Kates M. Technique of lipidology: isolation, analysis and identification of lipids. In: Burdon RH, ed. Laboratory Techniques in Biochemistry and Molecular Biology. New York, NY: Elsevier; 1986:100-278

8 Furimsky A, Vuong N, Xu H, et al. Percoll gradient-centrifuged capacitated mouse sperm have increased fertilizing ability and higher contents of sulfogalactosylglycerolipid and docosahexaenoic acid-containing phosphatidylcholine compared to washed capacitated mouse sperm. Biol Reprod 2005;72(3):574-583

9 Iida N, Toida T, Kushi Y, et al. A sulfated glucosylceramide from rat kidney. J Biol Chem 1989;264(10):5974-5980

10 Fiehn O, Wohlgemuth G, Scholz M, et al. Quality control for plant metabolomics: reporting MSI-compliant studies. Plant J 2008; 53(4):691-704

11 Faull KF, Anderson PJ, Barchas JD, Berger PA. Selected ion monitoring assay for biogenic amine metabolites and probenecid in human lumbar cerebrospinal fluid. J Chromatogr A 1979;163(4):337-349

12 Pulfer M, Murphy RC. Electrospray mass spectrometry of phospholipids. Mass Spectrom Rev 2003;22(5):332-364

13 Hutchins PM, Moore EE, Murphy RC. Electrospray MS/MS reveals extensive and nonspecific oxidation of cholesterol esters in human peripheral vascular lesions. J Lipid Res 2011;52(11):2070-2083

14 Leiker TJ, Barkley RM, Murphy RC. Analysis of diacylglycerol molecular species in cellular lipid extracts by normal-phase LCelectrospray mass spectrometry. Int J Mass Spectrom 2011;305(23):103-109

15 Murphy RC, Leiker TJ, Barkley RM. Glycerolipid and cholesterol ester analyses in biological samples by mass spectrometry. Biochim Biophys Acta 2011;1811(11):776-783

16 Hsu FF, Turk J. Characterization of phosphatidylinositol, phosphatidylinositol-4-phosphate, and phosphatidylinositol-4,5-bisphosphate by electrospray ionization tandem mass spectrometry: a mechanistic study. J Am Soc Mass Spectrom 2000;11(11):986-999

$17 \mathrm{Gu}$ M, Kerwin JL, Watts JD, Aebersold R. Ceramide profiling of complex lipid mixtures by electrospray ionization mass spectrometry. Anal Biochem 1997;244(2):347-356
18 Hsu FF, Turk J. Studies on phosphatidylserine by tandem quadrupole and multiple stage quadrupole ion-trap mass spectrometry with electrospray ionization: structural characterization and the fragmentation processes. J Am Soc Mass Spectrom 2005;16(9): 1510-1522

19 Murphy RC, Axelsen PH. Mass spectrometric analysis of long-chain lipids. Mass Spectrom Rev 2011;30(4):579-599

20 Hsu FF, Turk J. Electrospray ionization/tandem quadrupole mass spectrometric studies on phosphatidylcholines: the fragmentation processes. J Am Soc Mass Spectrom 2003;14(4):352-363

21 Brügger B, Erben G, Sandhoff R, Wieland FT, Lehmann WD. Quantitative analysis of biological membrane lipids at the low picomole level by nano-electrospray ionization tandem mass spectrometry. Proc Natl Acad Sci U S A 1997;94(6):2339-2344

22 Hsu FF, Bohrer A, Turk J. Electrospray ionization tandem mass spectrometric analysis of sulfatide. Determination of fragmentation patterns and characterization of molecular species expressed in brain and in pancreatic islets. Biochim Biophys Acta 1998; 1392(2-3):202-216

23 Duffin K, Obukowicz M, Raz A, Shieh JJ. Electrospray/tandem mass spectrometry for quantitative analysis of lipid remodeling in essential fatty acid deficient mice. Anal Biochem 2000;279(2): 179-188

24 Do TQ Moshkani S, Castillo P, et al. Lipids including cholesteryl linoleate and cholesteryl arachidonate contribute to the inherent antibacterial activity of human nasal fluid. J Immunol 2008; 181(6):4177-4187

25 Hunt AN, Postle AD. Mass spectrometry determination of endonuclear phospholipid composition and dynamics. Methods 2006; 39(2):104-111

26 Sherman WR, Ackermann KE, Bateman RH, Green BN, Lewis I. Mass-analysed ion kinetic energy spectra and B1E-B2 triple sector mass spectrometric analysis of phosphoinositides by fast atom bombardment. Biomed Mass Spectrom 1985;12(8):409-413

27 Liebisch G, Drobnik W, Reil M, et al. Quantitative measurement of different ceramide species from crude cellular extracts by electrospray ionization tandem mass spectrometry (ESI-MS/MS). J Lipid Res 1999;40(8):1539-1546

28 Marbois BN, Faull KF, Fluharty AL, Raval-Fernandes S, Rome LH. Analysis of sulfatide from rat cerebellum and multiple sclerosis white matter by negative ion electrospray mass spectrometry. Biochim Biophys Acta 2000;1484(1):59-70

29 Kongmanas K, Xu H, Yaghoubian A, et al. Seminolipid quantification by colorimetric assay and ESI-LC-MS/MS-multiple reaction monitoring: compensation of seminolipid expression in $\mathrm{Cgt}+\mathrm{I}-$ mice. J Lipid Res 2010;51(12):3548-3558

30 Bernhard W, Hoffmann S, Dombrowsky H, et al. Phosphatidylcholine molecular species in lung surfactant: composition in relation to respiratory rate and lung development. Am J Respir Cell Mol Biol 2001;25(6):725-731

31 Vance JE, Tasseva G. Formation and function of phosphatidylserine and phosphatidylethanolamine in mammalian cells. Biochim Biophys Acta 2013;1831(3):543-554

32 Zemski Berry KA, Turner WW, VanNieuwenhze MS, Murphy RC. Characterization of oxidized phosphatidylethanolamine derived from RAW 264.7 cells using 4-(dimethylamino)benzoic acid derivatives. Eur J Mass Spectrom (Chichester, Eng) 2010;16(3): 463-470

33 Zemski Berry KA, Murphy RC. Electrospray ionization tandem mass spectrometry of glycerophosphoethanolamine plasmalogen phospholipids. J Am Soc Mass Spectrom 2004;15(10):1499-1508

34 Gardocki ME, Jani N, Lopes JM. Phosphatidylinositol biosynthesis: biochemistry and regulation. Biochim Biophys Acta 2005;1735(2): 89-100

35 Takahashi T, Suzuki T. Role of sulfatide in normal and pathological cells and tissues. J Lipid Res 2012;53(8):1437-1450 